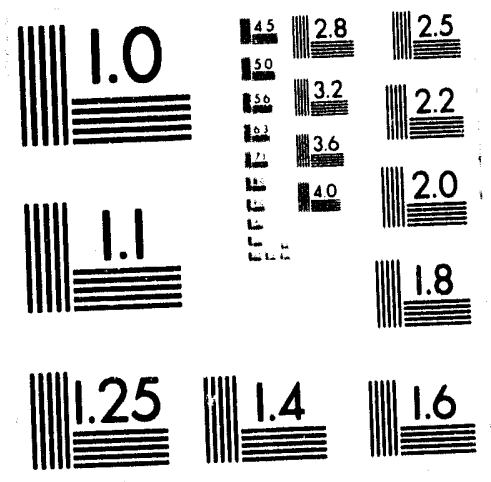



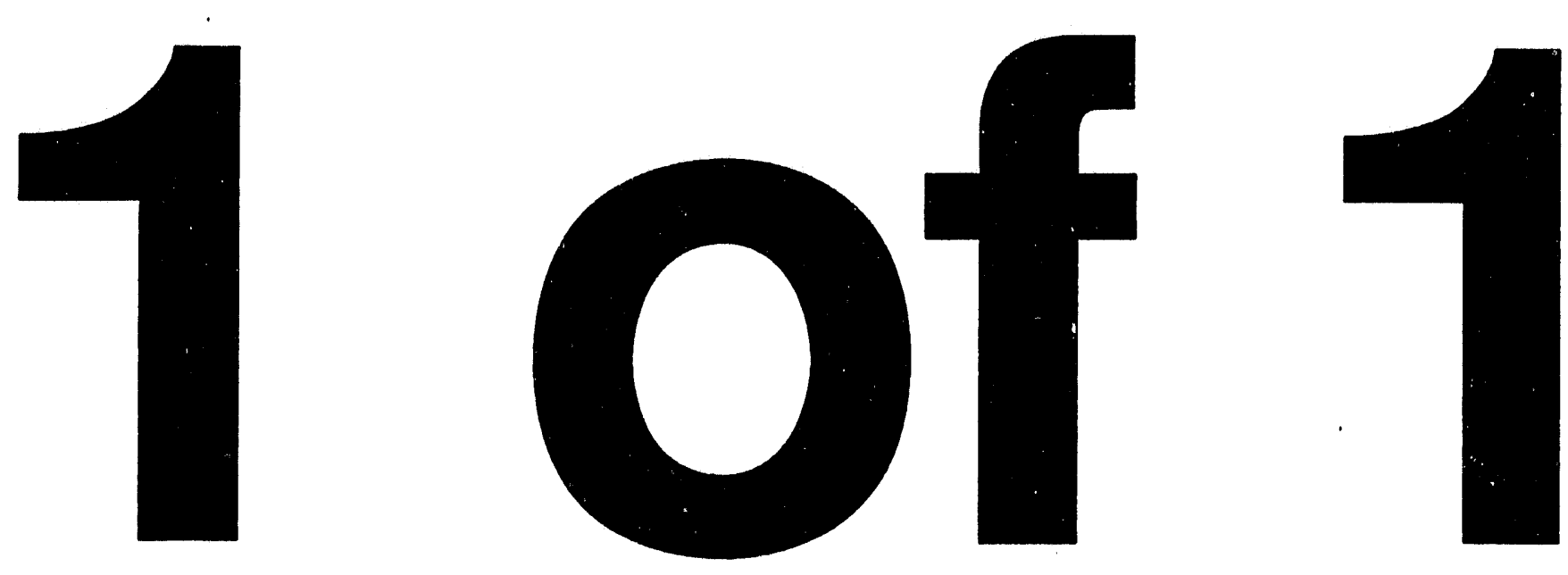
MONTE CARLO NEXT-EVENT POINT FLUX ESTIMATION FOR RCPOI

R. L. Martz

R. C. Gast

L. J. Tyburski

ANS Paper 


\section{NOTICE}

This report was prepared as an account of work sponsored by the United States Govenment. Neither the United States, nor the United States Department of Energy, nor any of their employees, nor any of their contractors, subcontractors, or their employees, makes any warranty, expressed or implied, or assumes any legal liability or responsibilty for the accuracy, completeness, or usefulness of any information, apparatus, product or process disclosed, or represents that its use would not infringe privately owned rights. 


\title{
MONTE CARLO NEXT-EVENT POINT FLUX ESTIMATION FOR RCPO1
}

R. L. Martz

R. C. Gast

L. J. Tyburski

\begin{abstract}
Two next event point estimators have been developed and programed into the RCPOI Monte Carlo program for solving neutron transport problems in three-dimensional geometry with detailed energy description. These estimators lise simplified but accurate flux-at-a-point tallying technique. Anisotropic scattering in the Lab system at the collision site is accounted for by determining the exit energy that corresponds to the angle between the location of the collision and the point detector. Elastic, inelastic, and thermal kernel scattering events ore included in this formulation. An averaging technique is used in both estimators to eliminate the well-known probiem of infinite variance due to collisions close to the point detector. In a novel approach to improve the estimator's efficiency, a Russian roulette scheme based on anticipated flux fall off is employed there averaging is not appropriate. A second estimator successfully uses a simple rejection technique in conjunction with detailed tracking where averaging isn't needed. Test results show good agreement with known numeric solutions. Efficiencies are examined as a function of input parameter selection and problem difficulty.
\end{abstract}

\section{INTRODUCTION}

Practical calculations of the flux due to a radiation source often require that the result be determined at a point. In an analog or normal mode Monte Carlo calculation, it is almost impossible to obtain a flux estimation by straight-forward techniques since no history can be expected to carry a particle through a given point. A time-honored alternative is to obtain an average over a finite volume surrounding the point of interest. This method may be satisfactory if the volume can be chosen small enough to adequately represent the flux at the point. As the gradient of the flux increases, the volume around the point of interest must decrease in order to preserve a true estimate of the flux at that point. However, the computer time required to generate a reasonable uncertainty increases as the volume decreases.

To circumvent this difficulty, an analytic statistical estimation of the flux at a point may be made. This technique is often referred to as a flux-at-apoint estimator or simply a point detector. A point detector is a deterministic estimate of the flux at a point in space and is made at source and collision events throughout the normal mode random walk. A point estimator is known as a next event estimator if a tally of the flux at a point is made when the next event is a trajectory without further collision directly to the point detector'. 
The chief difficulties with the next-event estimator in practical situations are: (1) The estimator requires computational effort due to geometric tracking. (2) An infinite variance problem is possible when a collision occurs near the point detector due to the $1 / R^{2}$ term in the analytical flux expression. In the past, authors ${ }^{1.4}$ have tried numerous approaches to eliminate or minimize these shortcomings. The approach of this work is to continue in this vein and to produce a simple but accurate point detector scheme which is efficient.

\section{BACKGROUNJ}

It can be shown ${ }^{1}$ that the general expression for the uncollided contribution to the flux at a point is given by

$$
\frac{f\left(\Omega^{\prime} \rightarrow \Omega\right)}{R^{2}} e^{-\lambda}
$$

where

$R$ is the distance to the detector from the collision or source point.

$f\left(\Omega^{\prime} \rightarrow \Omega\right)$ is the probability of scattering into the direction $\Omega$ from $\Omega^{\prime}$ or being born into the direction $\Omega$.
$\lambda=\int_{0}^{R} \Sigma_{t}(s) d s=\begin{aligned} & \text { total number of mean free paths integrated over the } \\ & \text { trajectory from the collision or source point to the }\end{aligned}$ point detector.

$s=$ measured distance along the direction from the collision or source point to the detector.

$\Sigma_{t}(s)=$ the total macroscopic cross section at $s$.

Except for special cases ${ }^{5}, f\left(\Omega^{\prime} \rightarrow \Omega\right)$ is a function of $\Omega \cdot \Omega^{\prime}=\mu_{0}$ only, where $\Omega^{\prime}$ and $\Omega$ are the directions before and after scattering, respectively, and $\mu_{0}$ is the cosine of the angle between them. The expression for the flux contribution to the detector becomes

$$
\phi=\frac{W_{e x}\left(\mu_{0}\right)}{4 \pi R^{2}} e^{-\lambda}
$$


where $W_{\text {ex }}$ is the exit neutron weight in the normal mode resulting from scattering at a collision site. It should be noted that $\lambda$ can only be obtained by actual tracking through the complicated geometry; consequently, this can be an expensive process.

Next-event estimation schemes often use the e ergy of the particle at the collision or source site in determining the flux contribution to the point detector. In reality, $\mu_{0}=1$, where $\mu_{0}$ is the cosine of the angle between the direction of the particle in the normal mode and the direction to the point detector, is extremely unlikely. Therefore, if the particle is to contribute to the flux at the point detector, it must undergo a fixed scatter from $\Omega^{\prime}$ to $\Omega$ and change its energy accordingly. The flux contribution, equation (2), to the point detector can be corrected by multiplying by the appropriate weight as follows

$$
\phi=\frac{W_{\text {ex }} W_{s c}}{4 \pi R^{2}} e^{-\lambda}
$$

where $W_{s c}$ is the weight scale factor to correct for the directional change from $\Omega$ to $\Omega$ as discussed above. $W_{s c}$ is determined from the actual scattering patterns for elastic, inelastic, and thermal kernel scattering. This method of correcting the energy at which the flux contribution is made to the point detector is used for the point estimator schemes discussed below.

\section{POINT ESTIMATION SCHEMES}

Two point estimators, having variations on the theme discussed above, were programmed into the RCPO1 code $^{6}$ and are presented in this paper. Estimator \#1 uses a flux averaging method similar to that of $M C N P^{1}$ where the flux inside a sphere of radius $R_{s 1}$ is given by

$$
\phi=\frac{3 W}{4 \pi R_{81}^{3} \Sigma_{T}(s)}\left(1-e^{\cdot \lambda}\right)
$$

where $W=W_{\text {ex }} W_{s c}$ and $R_{s 1}$ is a constant. This equation is based on the assumption that the scattering sites inside the sphere along the line between the collision site and the point detector are evenly distributed. Unlike equation (3), equation (4) will not lead to the infinite variance problem as $R \rightarrow \theta$. 
With the method proposed here, not only is the variance finite, but all moments exist for the frequency distribution function. Thus, the central limit theorem applies and the standard deviation on the flux estimate will decline as $\left(1 / N^{2}\right)$ where $N$ is the total number of histories.

Outside the sphere, Russian Roulette is played to reduce the amount of detailed geometry tracking. As part of the input for each point detector, the numbar of mean free paths (MFP) over which the flux is thought to decline by a factor of two must be specified. If the particle is outside the sphere,

$$
\text { let } n=\text { Int Part } \frac{\left(R-R_{s 1}\right) \Sigma_{T}(s)}{M F P} \text {. }
$$

Seiect a random number, $\rho$. If $\rho \leq 2^{\cdot n}$, set $W_{R}=2^{n}$; otherwise $W_{R}=0$.

If MFP is input as negative, Russian Roulette is not played outside the sphere and detailed geometry tracking is performed for every next event estimation. In this case or if $R<R_{s 1}, W_{R}=1$. The weight term, $W$, in the flux contribution expression is now defined as $W=W_{\text {ex }} W_{s c} W_{R}$ and the flux is scored as in Equation (3). With the correct choice of MFP a large kill off probability can be achieved for contributions remote from the detector, thus increasing the efficiency of the scheme by eliminating tracking of small contributors.

Estimator \#2 uses the flux averaging method as discussed with Estimator \#1. In addition, a second sphere is specified with a radius, $R_{s 2}$, which is larger than that of the first sphere. In the region that is outside of the first sphere but inside the second sphere, detailed geometry tracking is used for the point detector. For each source or scattering event outside the large sphere, the particle is considered to be moving to the detector with weight $W /\left(4 \pi R^{2}\right)$, where $W$ is the product of $W_{e x}$ and $W_{s c}$. A uniformly distributed random number, $\rho$, is drawn from which a test mean free path is computed from $D$ - $-\ln (\rho)$. If this falls short of the detector, tracking is terminated and no tally is made (i.e. the contribution is rejected). If the number of mean free paths selected goes beyond the detector, then $W /\left(4 \pi R^{2}\right)$ is tallied.

\section{VERIFICATION}

Initial test problems involved calculating the flux contribution from a point isotropic source in an infinite homogeneous medium at various distances. Accurate numerical solutions can be obtained?. The test cases presented here verify that the point estimation schemes are functioning properly and will give some insight into choosing some appropriate estimator parameters. 
In the limit as $R_{s 1}$ approaches zero, both schemes presented here exhibit behavior characteristic of the next event estimator (NEE). Figure 1 shows the cumulative point estimator flux result with associated $95 \%$ confidence interval statistical error bars versus batch number at the $0.5 \mathrm{~cm}$ location when the NEE approximation is used. The insert to figure 1 shows the corresponding relative statistical error as a function of batch number. Figure 2 shows similar plots for the NEE approximation at the $3.0 \mathrm{~cm}$ location. Figures 1 and 2 show the larger statistical error and irregular behavior that is characteristic of the NEE. This behavior is similar to results reported for the point estimation schemes of Reference (4). When Scheme $\# 2$ is used with $R_{s 2}=R_{s 1}=1.0 \mathrm{~cm}$ at the $3.0 \mathrm{~cm}$ location, Figure 3 shows how the results of Figure 2 can be improved.

\section{PARAMETER INSIGHT}

A concern of the analyst who uses these types of point estimators is what choice of parameters will yield an accurate result with the least amount of computer time. From the problem computation times and statistical uncertainties, Figures of Merit (FOM) can be calculated for each case. As the FOM increases so does the efficiency of the method. By running parametric studies with the point estimators and computing FOMs, insight for parameter selection can be established.

Table I presents a comparison of the results from the two point estimation methods discussed here. Examination of Table I reveals that as the spherical volume over which Equation (4) is applied increases, the statistical uncertainty decreases. But, if this spherical volume becomes too large, the volume weighted answer will deviate from the true result if the flux gradient is significant. This is as expected. For the simple cases presented, $R_{a 1}$ 's of 0.35 $\mathrm{cm}$ to $1.4 \mathrm{~cm}$ will yield results that are within $5 \%$ of the numeric for point detector locations of $0.5 \mathrm{~cm}$ to $5.0 \mathrm{~cm}$, respectively. In simple cases such as those studied here it may be possible to select a priori a value for $R_{s 1}$. In more complex source/geometry arrangements short test runs may be neederd to see how the flux varies with $R_{s i}$.

It is desirable to use the largest value of $R_{a 1}$ in order to maximize the FOM. It should be noted that in certain circumstances when a result near the source is needed, e.g. the $0.5 \mathrm{~cm}$ location, the largest $R_{\mathrm{s} 1}$ which will yield a result within a few percent of the known numeric is less than $1.0 \mathrm{~cm}$. In the region where $R_{s 1}<r<1.0$, Equation (3) will be used to determine the flux, thus, resulting in some large contributions. This will cause some irregularities in the variance. 


\section{COMPARISONS}

Cases were analyzed using Scheme \#1 with various choices for the MFP. When the MFP value was set to infinity, all contributions outside of the $R_{a 1}$ radius were followed. Significantly lower FOMs resulted due to increased tracking beyond $R_{81}$. An optimum choice for MFP was 1.0. Values lower than 1.0 were tried but no significant efficiency gains were noticed. For the cases presented in Table I, efficiency increases from $20 \%$ to $40 \%$ were noted when the MFP value was changed from infinity to 1.0 .

Cases were also analyzed using Scheme \#2 with various choices for $R_{81}$ and $R_{s 2}$. For point detector locations closer to the source, the FOM appears to be more sensitive to the ratio of $R_{82}$ to $R_{81}$. In the $0.5 \mathrm{~cm}$ location case where $R_{81}=$ $0.45 \mathrm{~cm}$, a $20 \%$ increase in the FOM is noted when $R_{82}$ is shrunk from $0.9 \mathrm{~cm}$ to $0.5 \mathrm{~cm}$. For point detector locations at distances of 3.0 and $5.0 \mathrm{~cm}$ from the source, the opposite observation is noted.

A comparison of FOMs for Schemes $\# 1$ and \#2 from Table I when $R_{\text {a1 }}$ is held constant shows that Scheme $\# 2$ can be as much as $20 \%$ more effictent than Scheme 11. As a further test for these two schemes, the simple homogeneous geometry used to generate Table I results was slightly complicated by placing five thin, fictitious assemblies between the point source and the detector at 0.5 $\mathrm{cm}$. The material of these assemblies was identical to that of the surrounding homogeneous material. This action introduced boundaries into the problem which are similar to those used in modeling more complicated problems.

Several cases were analyzed using the geometry with fictitious boundaries. The results of these cases are shown in Table II. A comparison of FOMs for the two schemes shows a more dramatic difference than is evident from a similar comparison of Table I results. There is almost a factor of $2.0 \mathrm{in}$ crease in efficiency when Scheme $\# 2$ is used. This difference is due to the way tracking is handled in the two schemes. The difference might be more or less in other geometric arrangements dependent upon number and location of boundaries.

\section{VOLUME AVERAGE RESULTS}

As stated in the introduction to this paper, a time-honored alternative to finding the flux at a point is to obtain an average over a firite volume surrounding the point of interest. For comparative purposes that method was used with a non-terminating collision estimator for a location of $5 \mathrm{~cm}$ from the source. Five different volumes ranging from $8 \mathrm{~cm}^{3}$ down to $0.027 \mathrm{~cm}^{3}$ were used. Results from these regions are presented in Table III. Only the result from the $8 \mathrm{~cm}^{3}$ region yields a FOM comparable to those of the point estimators of Table I. However, no result from this volume averaging method is within 
30\% of the known numeric. Therefore, the point estimator schemes presented here result in a marked improvement over what used to be the standard RCPO1 method for determining the flux in a very small volume.

\section{SUMMARY}

This work has presented two flux-at-a-point methods which sample from actual scattering patterns instead of assuming isotropic scattering and are fairly simple to implement. The well known infinite variance problem is handled by using a flux averaging method along a line inside a sphere of given radius around the point where the flux is to be determined. In the limit that this radius approaches zero, the classic next event estimation behavior is obtained.

Input parameter selection for the two schemes will be problem dependent. However, choices for these parameters can be determined from short, sample runs where the flux behavior and FOM are monitored as the input parameters are changed.

In the simple problems studied here, Scheme $\# 2$, which uses a simple rejection technique to eliminate unnecessary tracking outside the averaging sphere, is more efficient than Scheme 11 , which employs a Russian roulette method for the same purpose. This difference in efficiency increases when geometry boundaries are introduced.

The time-honored method of using volumetric averages to represent the flux at a point was demonstrated to be less accurate and generally less efficient than the point estimation methods of this work for the computation times used in this study.

\section{REFERENCES}

1) J. F. Briesmeister, ed., "MCNP -- A General Monte Carlo Code for Neutron and Photon Transport, Version 3A," LA-7396-M, Revision 2, 1986 (DE-87000708).

2) M. H. Kalos, "On the Estimation of Flux at a Point by Monte Carlo," Nucl. Sct. Eng, 16, 111 (1963).

3) H. A. Steinberg and M. H. Kalos, "Bounded Estimators for Flux at a Point in Monte Carlo," Nucl. Sci. Eng, 44, 406 (1971).

4) H. J. Kalli and E. D. Cashwell, "Evaluation of Three Monte Carlo Estimation Schemes for Flux at a Point," LA-6865-MS, September 1977. 
5) G. I. Bell and S. Glasstone, "Nuclear Reactor Theory," Robert E. Krieger Publishing Co., Malabar, Florida, 1979.

6) N. R. Candelore, R. C. Gast, and L. A. Ondis II, "RCPO1 .- A Monte Carlo Program for Solving Neutron and Photon Transport Problems in ThreeDimensional Geometry with Detailed Energy Description, "WAPD-TM-1267, August 1978.

7) K. M. Case, F. de Hoffmann, and G. Placzek, "Introduction to the Theory of Neutron Diffusion, "Los Alamos Scientific Laboratory, Los Alamos, New Mexico, June 1953.

8) J. Spanier and E. M. Gelbard, "Monte Carlo Principles and Neutron Transport Problems, " Addison-Wesley Publishing Company, Reading, Massachusetts, 1969. 
TABLE I

POINT ESTIMATOR COMPARISON

INFINITE HOMOGENEOUS MEDIUM

\begin{tabular}{|c|c|c|c|c|c|c|c|c|c|}
\hline METHOO & Rs 1 & $R s 2$ & MFP & LOCATION & FLUX & $95 \times$ C.1. & $\begin{array}{l}\text { TIME } \\
(\min )\end{array}$ & FOM & EXPECTED \\
\hline 1 & $\begin{array}{l}0.05 \\
0.10 \\
0.25 \\
0.25\end{array}$ & $\begin{array}{l}\ddot{*} \\
\ddot{*}\end{array}$ & $\begin{array}{l}1 \\
1 \\
1 \\
0\end{array}$ & $\begin{array}{l}0.5 \\
0.5 \\
0.5 \\
0.5\end{array}$ & $\begin{array}{l}5.125 E \cdot 02 \\
5.186 E \cdot 02 \\
5.297 E \cdot 02 \\
5.297 E \cdot 02\end{array}$ & $\begin{array}{l}1.602 E \cdot 03 \\
1.187 E \cdot 03 \\
8.668 E \cdot 04 \\
8.669 E \cdot 04\end{array}$ & $\begin{array}{l}11.5 \\
11.6 \\
11.7 \\
16.6\end{array}$ & $\begin{array}{r}339.5 \\
632.4 \\
1221.8 \\
861.4\end{array}$ & $\begin{array}{l}5.216 E \cdot 02 \\
5.216 E \cdot 02 \\
5.216 E \cdot 02 \\
5.216 E \cdot 02\end{array}$ \\
\hline 2 & $\begin{array}{l}0.18 \\
0.18 \\
0.25 \\
0.30 \\
0.30 \\
0.45 \\
0.45\end{array}$ & $\begin{array}{l}0.20 \\
0.90 \\
0.25 \\
0.50 \\
0.60 \\
0.50 \\
0.90\end{array}$ & $\begin{array}{l}\ddot{*} \\
\ddot{*} \\
\ddot{*} \\
\ddot{*}\end{array}$ & $\begin{array}{l}0.5 \\
0.5 \\
0.5 \\
0.5 \\
0.5 \\
0.5 \\
0.5\end{array}$ & $\begin{array}{l}5.189 E \cdot 02 \\
5.201 E \cdot 02 \\
5.299 E \cdot 02 \\
5.352 E \cdot 02 \\
5.355 E \cdot 02 \\
5.777 E \cdot 02 \\
5.776 E \cdot 02\end{array}$ & $\begin{array}{l}1.089 E \cdot 03 \\
1.109 E \cdot 03 \\
9.029 E \cdot 04 \\
8.754 E \cdot 04 \\
8.993 E \cdot 04 \\
5.524 E \cdot 04 \\
6.010 E \cdot 04\end{array}$ & $\begin{array}{l}9.0 \\
9.1 \\
9.0 \\
9.0 \\
9.1 \\
9.0 \\
9.1\end{array}$ & $\begin{array}{r}968.6 \\
926.9 \\
1463.1 \\
1581.5 \\
1502.1 \\
4655.4 \\
3891.2\end{array}$ & $\begin{array}{l}5.216 E \cdot 02 \\
5.216 E \cdot 02 \\
5.216 E \cdot 02 \\
5.216 E \cdot 02 \\
5.216 E \cdot 02 \\
5.216 E \cdot 02 \\
5.216 E \cdot 02\end{array}$ \\
\hline 1 & $\begin{array}{l}0.25 \\
0.50 \\
0.50 \\
0.90 \\
1.00\end{array}$ & $\begin{array}{l}\ddot{.} \\
\ddot{.} \\
\ddot{.}\end{array}$ & $\begin{array}{l}1 \\
1 \\
0 \\
1\end{array}$ & $\begin{array}{l}3.0 \\
3.0 \\
3.0 \\
3.0 \\
3.0\end{array}$ & $\begin{array}{l}5.122 E \cdot 04 \\
4.840 E \cdot 04 \\
4.826 E \cdot 04 \\
4.916 E \cdot 04 \\
4.880 E \cdot 04\end{array}$ & $\begin{array}{l}8.360 E \cdot 05 \\
2.946 E \cdot 05 \\
2.923 E \cdot 05 \\
1.780 E \cdot 05 \\
1.693 E \cdot 05\end{array}$ & $\begin{array}{l}17.2 \\
12.0 \\
16.6 \\
16.6 \\
11.9\end{array}$ & $\begin{array}{r}8.4 \\
86.5 \\
63 \\
176.5 \\
268.4\end{array}$ & $\begin{array}{l}4.766 E \cdot 04 \\
4.766 E \cdot 04 \\
4.766 E \cdot 04 \\
4.766 E \cdot 04 \\
4.766 E \cdot 04\end{array}$ \\
\hline 2 & $\begin{array}{l}0.25 \\
0.45 \\
0.45 \\
0.63 \\
0.63 \\
1.00 \\
1.00\end{array}$ & $\begin{array}{l}0.25 \\
0.50 \\
0.90 \\
0.70 \\
0.90 \\
1.00 \\
2.00\end{array}$ & 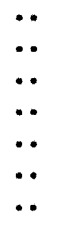 & $\begin{array}{l}3.0 \\
3.0 \\
3.0 \\
3.0 \\
3.0 \\
3.0 \\
3.0\end{array}$ & $\begin{array}{l}4.928 E \cdot 04 \\
4.793 E \cdot 04 \\
4.795 E \cdot 04 \\
4.818 E \cdot 04 \\
4.802 E \cdot 04 \\
4.919 E \cdot 04 \\
4.963 E \cdot 04\end{array}$ & $\begin{array}{l}7.183 E \cdot 05 \\
3.626 E \cdot 05 \\
3.699 E \cdot 05 \\
2.933 E \cdot 05 \\
2.807 E \cdot 05 \\
2.034 E \cdot 05 \\
1.905 E \cdot 05\end{array}$ & $\begin{array}{l}9.0 \\
9.0 \\
9.1 \\
9.4 \\
9.1 \\
9.4 \\
9.4\end{array}$ & $\begin{array}{r}20 \\
74.2 \\
73.1 \\
110.4 \\
123.8 \\
239.4 \\
277.9\end{array}$ & $\begin{array}{l}4.766 E \cdot 04 \\
4.766 E \cdot 04 \\
4.766 E \cdot 04 \\
4.766 E \cdot 04 \\
4.766 E \cdot 04 \\
4.766 E \cdot 04 \\
4.766 E \cdot 04\end{array}$ \\
\hline 1 & $\begin{array}{l}1.00 \\
1.00\end{array}$ & $\ddot{.}$ & 1 & $\begin{array}{l}5.0 \\
5.0\end{array}$ & $\begin{array}{l}3.471 E \cdot 05 \\
3.492 E \cdot 05\end{array}$ & $\begin{array}{l}2.458 E \cdot 06 \\
2.943 E \cdot 06\end{array}$ & $\begin{array}{l}61.6 \\
56.2\end{array}$ & $\begin{array}{r}12.4 \\
9.6\end{array}$ & $\begin{array}{l}3.362 E \cdot 05 \\
3.362 E \cdot 05\end{array}$ \\
\hline 2 & $\begin{array}{l}1.00 \\
1.00\end{array}$ & $\begin{array}{l}1.00 \\
2.00\end{array}$ & $\ddot{*}$ & $\begin{array}{l}5.0 \\
5.0\end{array}$ & $\begin{array}{l}3.438 E \cdot 05 \\
3.476 E \cdot 05\end{array}$ & $\begin{array}{l}2.899 E \cdot 06 \\
2.482 E \cdot 06\end{array}$ & $\begin{array}{l}44.7 \\
59.6\end{array}$ & $\begin{array}{l}12.1 \\
12.6\end{array}$ & $\begin{array}{l}3.362 E \cdot 05 \\
3.362 E \cdot 05\end{array}$ \\
\hline
\end{tabular}

INFINITE HOMOGENEOUS MEDIUM WITH FICTIIIOUS BOUNOARIES

\begin{tabular}{|c|c|c|c|c|c|c|c|c|c|}
\hline METHOD & $R \& 1$ & R82 & MFP & LOCATION & FLUX & $95 \times<.1$. & $\begin{array}{l}\text { TIME } \\
\text { (min) }\end{array}$ & FOM & EXPECTED \\
\hline 1 & $\begin{array}{l}0.25 \\
0.25\end{array}$ & $\ddot{*}$ & $i$ & $\begin{array}{l}0.5 \\
0.5\end{array}$ & $\begin{array}{l}5.264 E \cdot 02 \\
5.251 E \cdot 02\end{array}$ & $\begin{array}{l}1.777 \mathrm{E} \cdot 03 \\
1.052 \mathrm{E} \cdot 03\end{array}$ & $\begin{array}{l}18.3 \\
17.2\end{array}$ & $\begin{array}{l}183.3 \\
557.1\end{array}$ & $\begin{array}{l}5.216 E \cdot 02 \\
5.216 E \cdot 02\end{array}$ \\
\hline 2 & $\begin{array}{l}0.25 \\
0.25\end{array}$ & $\begin{array}{l}0.5 \\
1.0\end{array}$ & $\ddot{~}$ & $\begin{array}{l}0.5 \\
0.5\end{array}$ & $\begin{array}{l}5.274 E \cdot 02 \\
5.274 E \cdot 02\end{array}$ & $\begin{array}{l}9.515 E \cdot 04 \\
1.006 E \cdot 03\end{array}$ & $\begin{array}{l}12.3 \\
13.2\end{array}$ & $\begin{array}{l}959.7 \\
802.1\end{array}$ & $\begin{array}{l}5.216 E \cdot 02 \\
5.216 E \cdot 02\end{array}$ \\
\hline 1 & $\begin{array}{l}0.25 \\
0.25 \\
0.50 \\
0.50\end{array}$ & $\begin{array}{l}\ddot{.} \\
\ddot{0} \\
\ddot{*}\end{array}$ & $\begin{array}{l}0 \\
1 \\
0 \\
1\end{array}$ & $\begin{array}{l}3.0 \\
\mathbf{3 . 0} \\
\mathbf{3 . 0} \\
\mathbf{3 . 0}\end{array}$ & $\begin{array}{l}4.788 E \cdot 04 \\
5.122 E \cdot 04 \\
4.740 E \cdot 04 \\
4.802 E \cdot 04\end{array}$ & $\begin{array}{l}1.331 E \cdot 04 \\
8.360 E \cdot 05 \\
8.292 E \cdot 05 \\
4.122 E \cdot 05\end{array}$ & $\begin{array}{l}18.1 \\
17.2 \\
18.3 \\
18.4\end{array}$ & $\begin{array}{r}2.7 \\
8.4 \\
6.9 \\
28.4\end{array}$ & $\begin{array}{l}4.766 E \cdot 04 \\
4.766 E \cdot 04 \\
4.766 E \cdot 04 \\
4.766 E \cdot 04\end{array}$ \\
\hline 2 & $\begin{array}{l}0.50 \\
0.50 \\
0.50\end{array}$ & $\begin{array}{l}0.5 \\
1.0 \\
2.0\end{array}$ & $\begin{array}{l}\ddot{.} \\
\ddot{*}\end{array}$ & $\begin{array}{l}3.0 \\
3.0 \\
3.0\end{array}$ & $\begin{array}{l}4.874 E \cdot 04 \\
4.870 E \cdot 04 \\
4.712 E \cdot 04\end{array}$ & $\begin{array}{l}4.842 E \cdot 05 \\
4.714 E \cdot 05 \\
4.993 E \cdot 05\end{array}$ & $\begin{array}{l}8.8 \\
9.4 \\
9.6\end{array}$ & $\begin{array}{l}44.4 \\
43.7 \\
35.7\end{array}$ & $\begin{array}{l}4.766 E \cdot 04 \\
4.766 E \cdot 04 \\
4.766 E \cdot 04\end{array}$ \\
\hline
\end{tabular}

VOLUME AVERAGE COMPARISON

\begin{tabular}{|c|c|c|c|c|c|}
\hline $\begin{array}{l}\text { VOLUNE } \\
\text { (CC) }\end{array}$ & $\begin{array}{l}\text { AVERAGE } \\
\text { FLUX }\end{array}$ & $5 x$ c. 1. & $\begin{array}{l}\text { TIME } \\
\text { (MIN) }\end{array}$ & Fon & XPECTEO \\
\hline $\begin{array}{l}8.000 \\
1.000 \\
0.125 \\
0.064 \\
0.027\end{array}$ & $\begin{array}{l}6.687 E \cdot 05 \\
6.380 E \cdot 05 \\
4.727 E \cdot 05 \\
2.246 E \cdot 05 \\
2.660 E \cdot 05\end{array}$ & $\begin{array}{l}4.883 E \cdot 06 \\
1.219 E-05 \\
1.654 E \cdot 05 \\
5.805 E \cdot 06 \\
1.034 E \cdot 05\end{array}$ & $\begin{array}{l}59.6 \\
59.6 \\
59.6 \\
59.6 \\
59.6\end{array}$ & $\begin{array}{r}12.10 \\
1.80 \\
0.53 \\
0.96 \\
0.43\end{array}$ & $\begin{array}{l}3.362 E \cdot 05 \\
3.362 E \cdot 05 \\
3.362 E \cdot 05 \\
3.362 E \cdot 05\end{array}$ \\
\hline
\end{tabular}


FIGURE 1

NEUTRON FLUX WITH ERROR BARS USING NEXT EVENT ESTIMATOR APPROXIMATION AT THE O.5 CM LOCATION

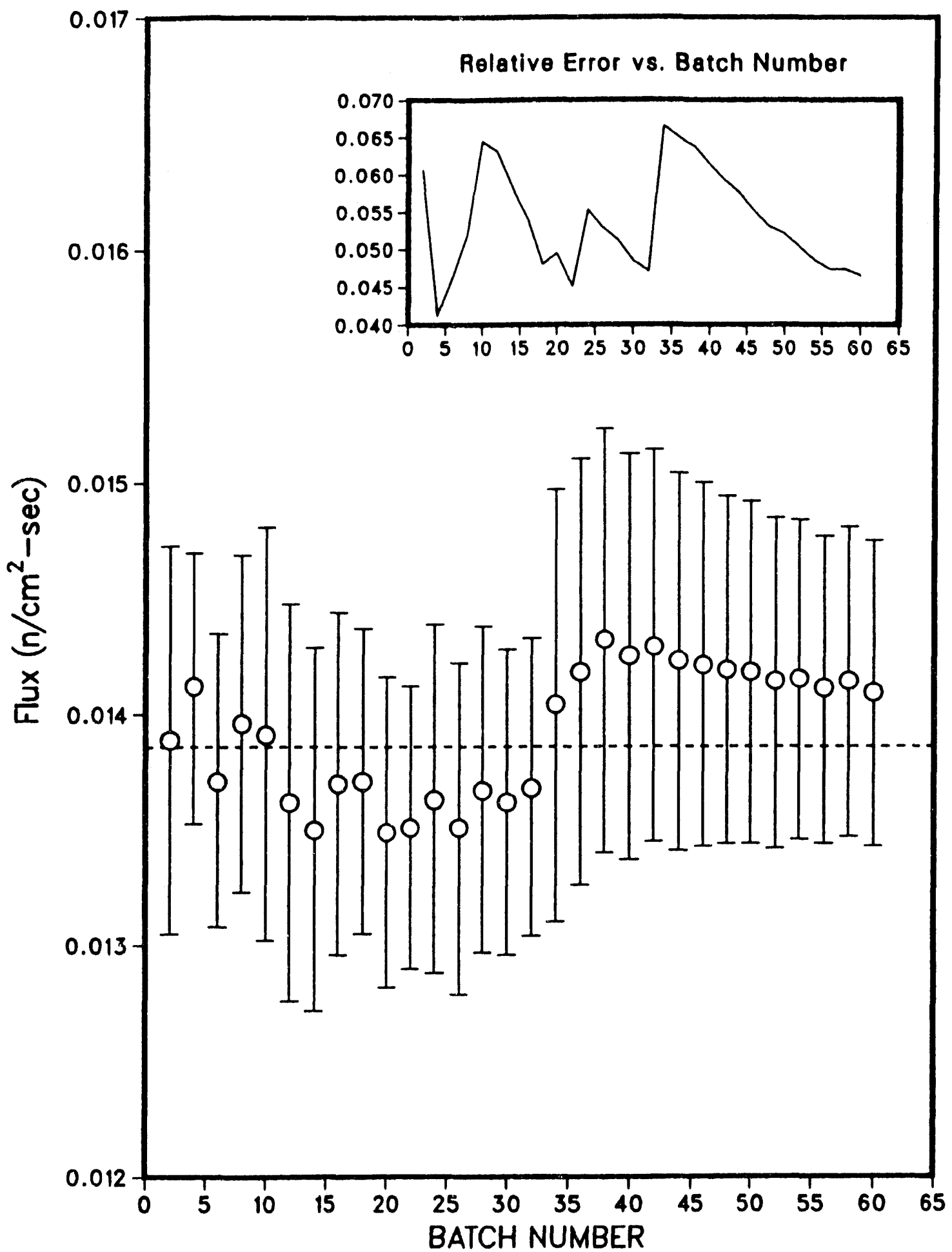


FIGURE 2

NEUTRON FLUX WITH ERROR BARS USING NEXT EVENT ESTIMATOR APPROXIMATION AT THE 3.0 CM LOCATION

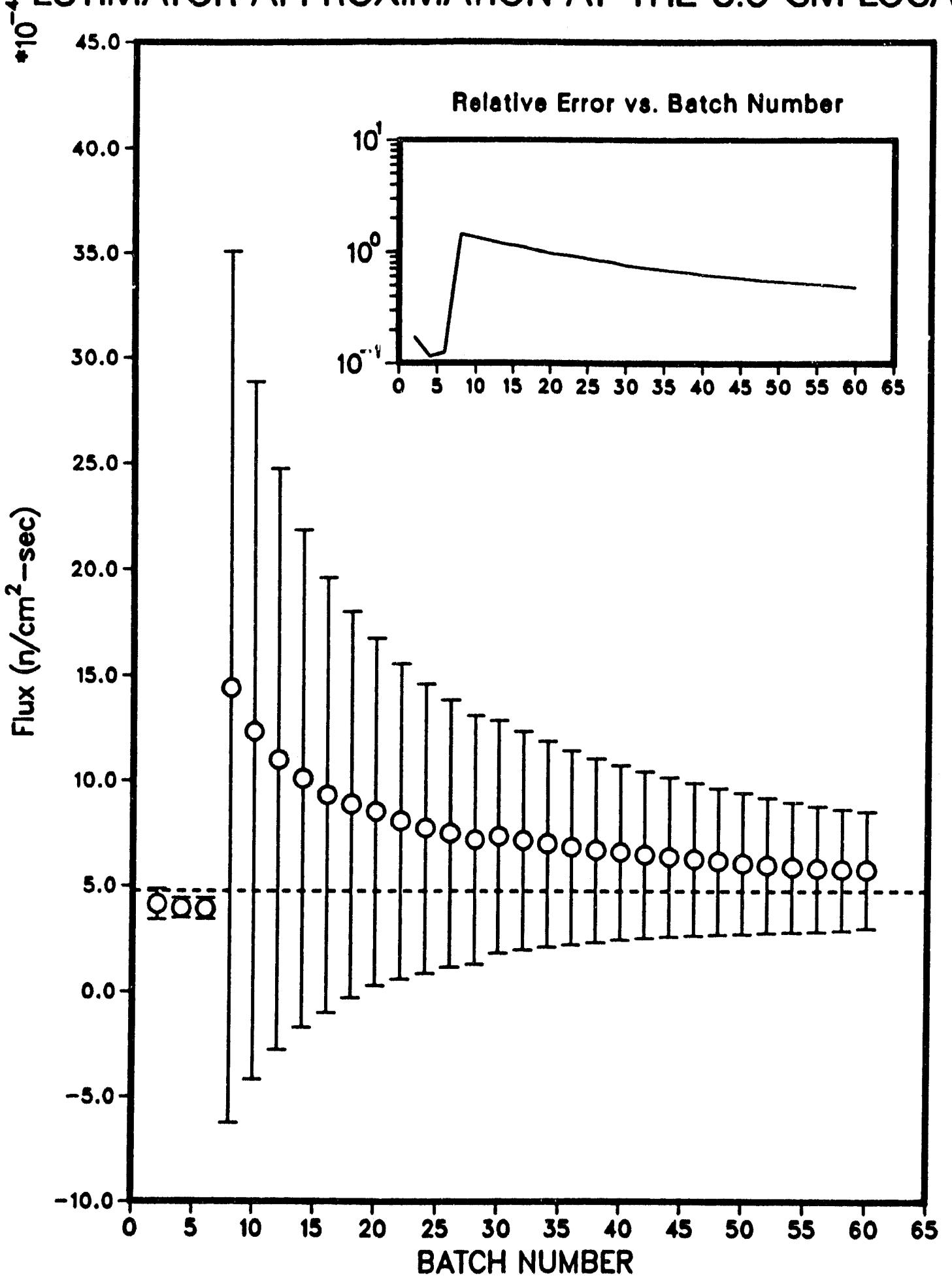


FIGURE 3

NEUTRON FLUX WITH ERROR BARS USING SCHEME \#2 WITH $R_{s 1}=R_{s 2}=1.0$ CM AT THE 3.0 CM LOCATION

1o

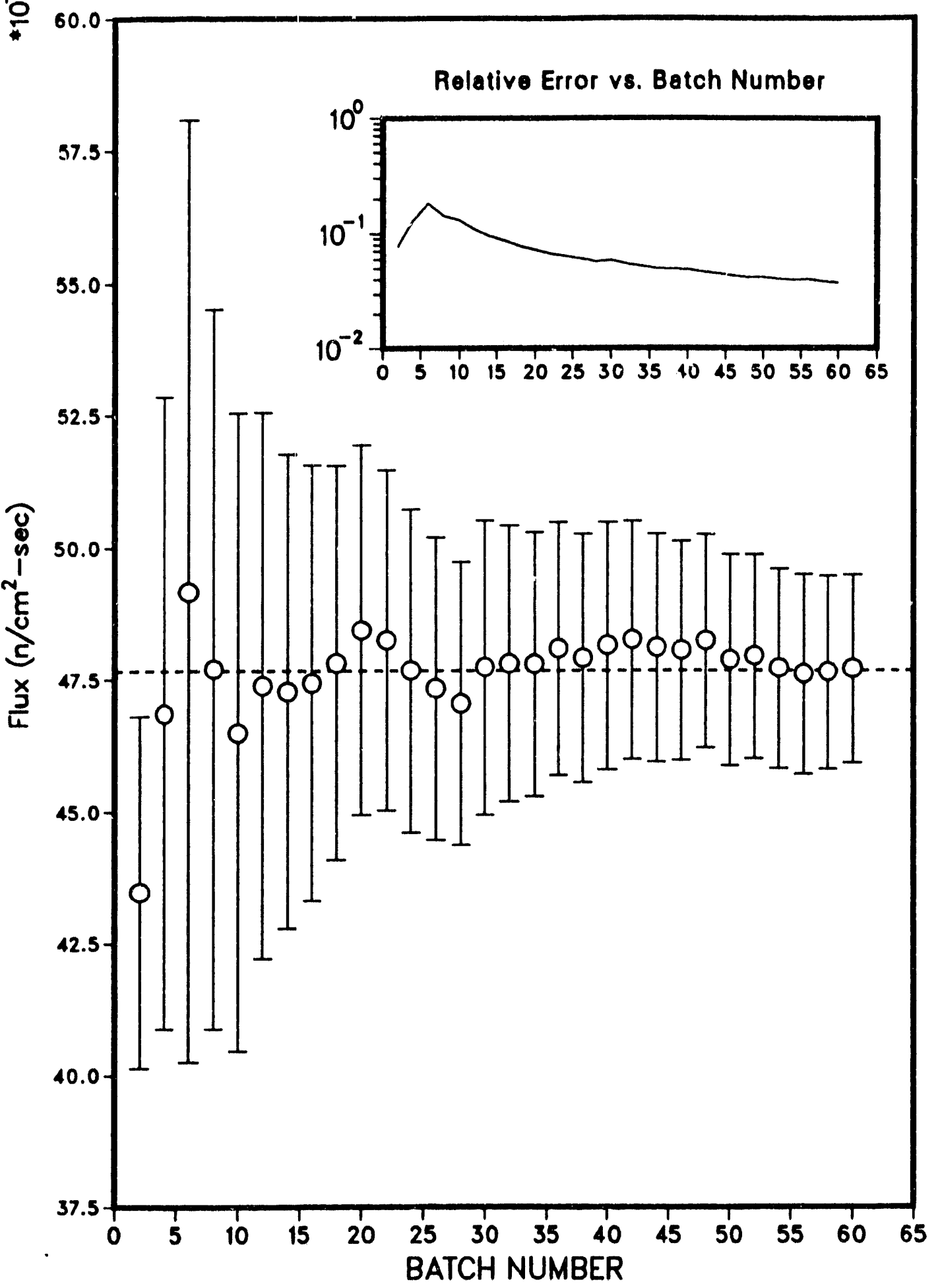



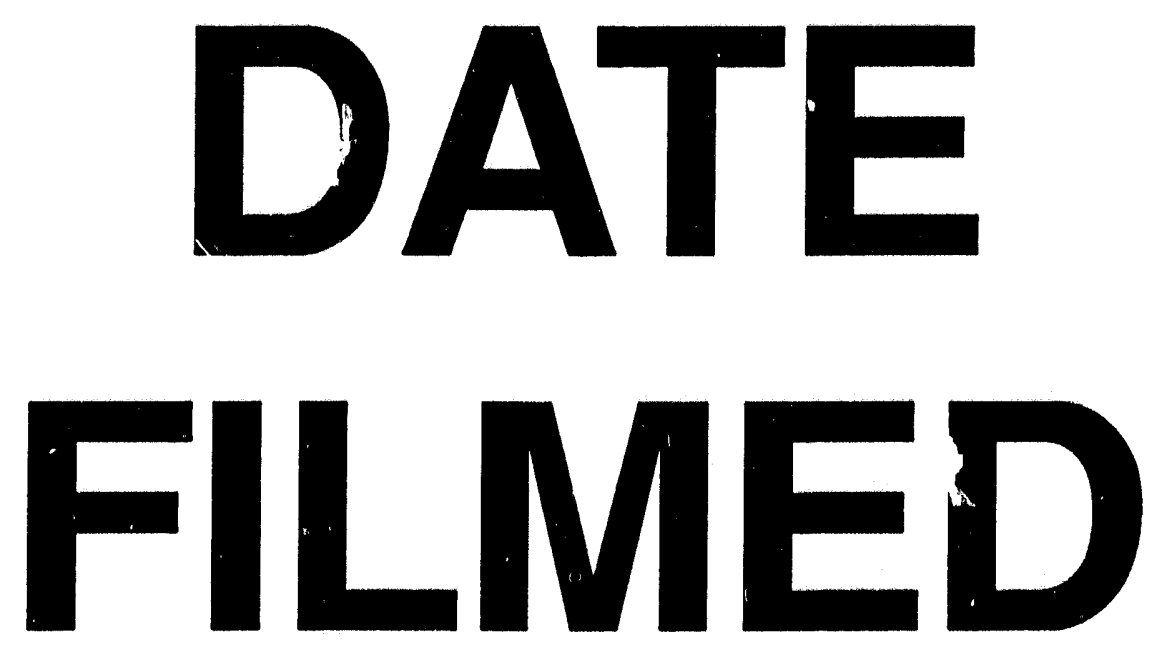

$6 / 10 / 94$
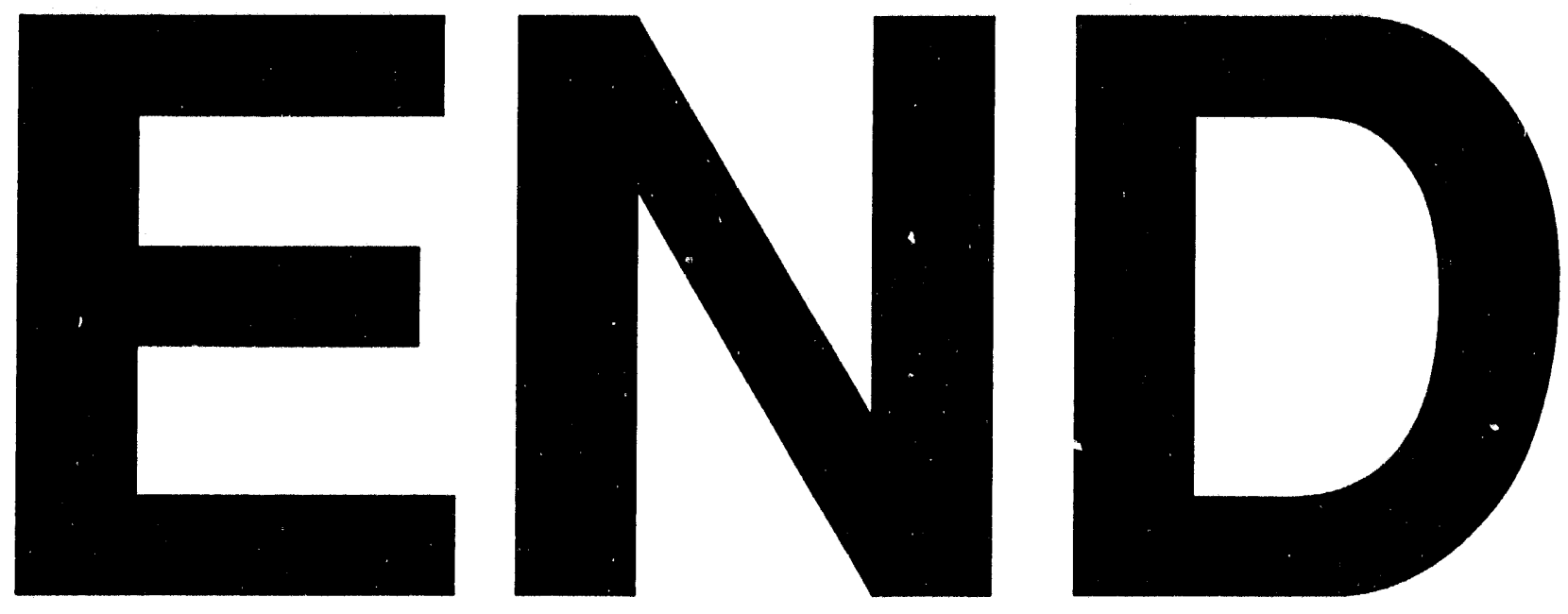
